\title{
Laços de afeto: as repercussões do estilo de apego primário e estabelecido entre casais no ajustamento conjugal
}

\author{
Ties Between Loved Ones: The Repercussions of the Primary fttachment Style \\ and the fttachment Style Established between Couples in the Marital fdjustment \\ Lazos de afecto: las repercusiones del estilo de apego primario y establecido \\ entre parejas en el ajuste conyugal
}

\author{
Natana Consoli* \\ Jade Wagner Bernardes* \\ Angela Helena Marin* \\ *Universidade do Vale do Rio dos Sinos, São Leopoldo, Brasil.
}

Doi: http://dx.doi.org/10.12804/revistas.urosario.edu.co/apl/a.5409

\section{Resumo}

O padrão de apego estabelecido na infância entre a criança e seu cuidador tende a se repetir nas relações futuras, como na conjugalidade, podendo facilitar ou dificultar tal relação. Nesse sentido, investigou-se a relação entre o padrão de apego primário e o padrão de apego estabelecido entre casais, e sua influência no ajustamento conjugal por meio de um delineamento comparativo, de corte transversal e abordagem quantitativa. Participaram 100 casais heterossexuais, que responderam a uma Ficha de Contato Inicial, ao Questionário de Vinculação ao Pai e a Mãe, à Escala de Experiência em Relações Próximas e à Escala Revisada de Ajustamento Conjugal. Os resultados indicaram a associação entre o padrão de apego primário seguro e menores níveis de evitação entre o casal e a relacionamentos bem ajustados e sem sofrimento, enquanto os padrões preocupado e desinvestido se relacionaram a maiores níveis de evitação e a relacionamentos desajustados e com sofrimento. Os dados revelam características importantes da dinâmica conjugal, que podem fundamentar a terapia de casais com vistas ao desenvolvimento de laços de afeto que contribuam para o bem-estar conjugal.

Palavras-chave: comportamento de apego, relações de objeto, relações conjugais.

\section{fibstract}

The attachment pattern established in childhood between the child and his caregiver tends to be repeated in future relationships, as in conjugality, and may facilitate or

* Natana Consoli, Jade Wagner Bernardes, Angela Helena Marin, Programa de Pós-graduação em Psicologia, Universidade do Vale do Rio dos Sinos, São Leopoldo, Brasil.

Correspondência a respeito deste artigo deve ser endereçada para Angela Helena Marin, Av. Unisinos, 950. Sala E01-109. Bairro Cristo Rei - São Leopoldo/RS. Correio eletrônico: angelahm@unisinos.br

Cómo citar este artículo: Consoli, N., Wagner Bernardes, J. \& Marin, A. H. (2018). Laços de afeto: as repercussões do estilo de apego primário e estabelecido entre casais no ajustamento conjugal. Avances en Psicología Latinoamericana, 36(2), 315-329. DOI: http://dx.doi.org/10.12804/revistas.urosario.edu.co/apl/a.5409 
hinder such relationship. In this sense, a research of the relationship between the primary pattern of attachment, the pattern of commitment established in the conjugal relationship, and the influence of these in the marital adjustment was conduced based on a comparative, quantitative and transversal study. A sample of 100 couples was used, they responded to an initial contact form, the father/mother attachment questionnaire, questions the experiences in close relationships and a dyadic adjustment scale revised. The results indicate that the primary insurance pattern was associated with lower levels of avoidance, between the couple well adjusted relation and no suffering; while the concerned was associated without investment attachment patterns, correlated with greater levels of avoidance, and with dysfunctional relationships and suffering. The data reveal important features of couple dynamics, which can be helped by couple's therapy for developing bonds of affection that contribute to the well-being of the couple.

Keywords: attachment behavior, object relations, marital relations.

\section{Resumen}

El patrón de apego establecido en la infancia tiende a repetirse en las relaciones futuras, como en la conyugalidad, pudiendo facilitar o dificultar tal relación. En ese sentido, se investigó la relación entre el patrón de apego primario y el patrón de unión que se establece entre las parejas y su influencia en el ajuste marital por medio de un diseño transversal y comparativa con enfoque cuantitativo. Participaron 100 parejas heterosexuales que respondieron el Formulario de Contacto Primero, el Cuestionario de Vinculación con el Padre y la Madre, la Escala para evaluar la Experiencia de Relaciones Cercanas y la Escala de Ajuste Conyugal. Los resultados indicaron la asociación entre el patrón de apego primario seguro y menores niveles de evitación entre la pareja y las relaciones bien ajustadas y sin sufrimiento, mientras que los patrones preocupados y desinvestidos se relacionaron con mayores niveles de evitación y relaciones desajustadas y con sufrimiento. Los datos revelan características importantes de la dinámica conyugal, que pueden fundamentar la terapia de parejas con vistas al desarrollo de lazos de afecto que contribuyan al bienestar conyugal.

Palabras clave: conducta de apego, relaciones de objeto, relaciones conyugales.

É sabido que o padrão de apego construído nas primeiras relações afetivas da infância tende a se manter nas demais relações estabelecidas ao longo da vida (Bowlby, 1989; Klaus, Kennel $\&$ Klaus, 2000). Dessa forma, sustenta-se que a continuidade do padrão relacional, mediada pelo desenvolvimento de modelos de apego, tende a guiar as interações sociais e o estabelecimento de relacionamentos futuros (Bowlby, 1989).

Conforme Bowlby (1969, 2009), o apego primário é um tipo de vínculo social estabelecido na relação entre a criança e seu cuidador principal, o qual pode ser observado por meio de comportamentos (chorar, fazer contato visual, tocar e agarrar-se) que possibilitam manter proximidade entre eles. Este pode ser classificado em três categorias, segundo Ainsworth, Blehar, Waters e Wall (1978): seguro, inseguro evitativo e inseguro ansioso-ambivalente. A criança com apego seguro percebe a figura do cuidador como disponível e responsiva, isto é, a considera como alguém que responde de forma adequada às suas necessidades (Scheeren, Delatorre, Neumann \& Wagner, 2015). Já a criança com apego inseguro evitativo, percebe que em situações de estresse, o cuidador não estará disponível emocionalmente, o que o leva a evitar o contato com ele (Irish \& Cassidy, 1997). Por fim, a criança com apego inseguro ansioso-ambivalente percebe que diante das situações-problema, o cuidado e a responsividade do cuidador é inconsistente, ou seja, ela não sabe se poderá contar com o suporte necessário, pois seu cuidador não está claramente disponível (Sroufe, Egelend, Carlson \& Collins, 2005).

O modo como se configuram as primeiras relações tende a funcionar como filtros utilizados para 
criar expectativas que influenciam nas escolhas pessoais, ações e relacionamentos que venham a se estabelecer em diversos contextos, como familiar, conjugal, profissional ou social (Montoro, 2004), com vistas a suprir as necessidades básicas de proteção. Attili (2006, p. 56), afirma que:

Os adultos, da mesma forma que as crianças, têm necessidade de que alguém não os perca de vista, cuide deles quando estão doentes, conforte-os quando estão abatidos, acalme-os na aflição e os aqueça a noite. Isso vale tanto para homens quanto para mulheres.

A repetição do padrão relacional estabelecido na infância nos demais relacionamentos tem sido atribuída ao que foi conceituado como modelo interno de trabalho (internal working model; Bowlby, 1980). Tal modelo é constituído pelas representações mentais e expectativas sobre o ambiente e as figuras de apego, além das estruturas cognitivas que orientam as interações ao longo da infância e das regras que são internalizadas (Ainsworth, 1989; Bartholomew, 1990; Pietromonaco \& Barrett 2000). Contudo, mesmo que o padrão de apego primário se mantenha ativo, tendendo a se atualizar e repetir ao longo da vida (Bowlby, 1989; Furman, Simon, Shaffer \& Bouchey, 2002), mudanças são esperadas uma vez que relações subsequentes podem atuar sobre esses modelos internos, alterando a visão do indivíduo sobre si e os outros (Bowlby, 1989; Fraley, 2002; Moreira, Martins, Gouveia \& Canavarro, 2015; Pietromonaco \& Barrett, 2000). Nesse sentido, faz-se relevante estudar as relações conjugais.

Ao investigar se a capacidade de amar e ser amado está relacionada com os vínculos de apego construídos quando criança, Lima (2010) apontou que a escolha do cônjuge ocorre a partir das influências dos padrões de apego, isto é, projeta-se no outro o que desde criança serviu como um modelo de identificação. De forma semelhante, Crowell, Treboux \& Waters (2002), a partir de uma pesquisa com casais americanos e hispânicos, utilizando uma experiência de resolução de problemas e uma escala para classificação de apego, constataram que os adultos com padrão de apego seguro apresentaram as mesmas categorias de comportamento que usariam na infância com suas figuras de apego primárias para a resolução de problemas com seu parceiro.

Para além da repetição dos padrões de apego primário nas relações, também há evidências de sua associação com a satisfação conjugal (Mosmann, Wagner \& Féres-Carneiro, 2006). Definir uma relação conjugal satisfatória tem sido complexo, devido à pluralidade de conceituações disponíveis na literatura nacional e internacional (Mosmann et al., 2006; Rosado \& Wagner, 2015; Scorsolini-Comin \& Santos, 2011). Portanto, neste estudo optou-se por utilizar o conceito de ajustamento diádico, definido a partir da comparação entre expectativas sobre a relação e seus resultados, que se refletem em um processo avaliado por meio da comunicação, felicidade, integração e satisfação (Spainer, 1976).

Pesquisas têm evidenciado que adultos com apego seguro tendem a sentir-se menos ameaçados em discussões com o parceiro, apresentam menor incidência de conflitos e maior uso de negociação, quando comparados a adultos com apego inseguro (Salvatore et al., 2011; Pistole \& Arricale, 2003; Scheeren et al., 2015). Montoro (2004) constatou que adultos com apego seguro tendem a ter mais equilíbrio entre proximidade e autonomia nas relações conjugais, maior facilidade e desejo de intimidade, pouca preocupação com o abandono, relações mais duradouras, boa impressão do outro e boa autoestima. Por outro lado, adultos com padrão de apego inseguro evitativo apresentam medo e desconforto com a intimidade, dão pouca atenção às experiências e necessidades do parceiro, além de expressarem necessidade de manter distância, mostrando-se mais inclinados a usar estilos negativos na resolução de conflitos (Bogda \& Sendil, 2012; Fisher \& Crandell, 2001). Já os adultos com apego inseguro ansioso-ambivalente, tendem a ter preocupação com a falta de proximidade, medo de 
rejeição e desejo constante de união. A intimidade tende a prevalecer sobre a autonomia, havendo ciúme extremo e submissão para ser aceito. Eles apresentam comumente autoconceito negativo e tendem a reagir com emoções exageradas em situação de ameaça, devido ao medo de perder o parceiro (Wang, King \& Debernardi, 2012), expressando e experimentando sentimentos intensos de mágoa durante os conflitos (Overall, Girme, Lemay \& Hammond, 2014).

Apesar destas constatações, Hazan \& Shaver (1987) indicaram que os indivíduos se utilizam dos modelos representacionais de si, mas também dos outros, para orientar suas relações amorosas, buscando explicar as diferenças individuais emocionais, comportamentais, cognitivas e de expectativas sobre tais relações. Collins \& Read (1990) estudaram os espaços compartilhados e individuais existentes entre os membros do casal (capacidade de compartilhar, habilidade individual e conjunta de desejar, disposição da necessidade de dependência entre os membros do casal, intensidade ou ausência da procura pelo reconhecimento do outro e características do apego que o casal cria conjuntamente) e constataram que o apego desenvolvido entre o casal pode funcionar como uma base segura, mesmo que os padrões de apego primário sejam inseguros, implicando em maior satisfação conjugal.

Frente ao exposto, diversos pesquisadores têm defendido a necessidade de novas pesquisas que visem uma melhor compreensão do desenvolvimento e construção das relações de apego ao longo da vida (Bretherton \& Munhollan, 2008; Dalben \& Dell'Aglio, 2005; Fraley, Waller \& Brennan, 2000). Além disso, destaca-se a importância da temática no âmbito da clínica psicológica com casais, uma vez que saber entender a repercussão que os padrões de apego primário têm sobre cada um dos cônjuges, pode auxiliar no manejo da dinâmica relacional, contribuindo para o estabelecimento de um padrão de apego que possa prover segurança para suprir as carências de um apego primário inseguro evitativo ou inseguro ansioso-ambivalente (Orbach, 2007), o que tende a favorecer o ajustamento conjugal. Nesse sentido, o objetivo do presente estudo foi investigar a relação entre o padrão de apego primário e o padrão de apego estabelecido entre o casal, bem como a influência destes no ajustamento conjugal. Como objetivos específicos buscou-se examinar as relações de apego primário de ambos os cônjuges, a relação entre o apego primário e o apego que se estabelece na relação conjugal, a relação do apego estabelecido entre o casal e a satisfação conjugal, e se há diferença entre homens e mulheres quanto ao padrão de apego estabelecido na relação e no ajustamento conjugal.

\section{Método}

\section{Delineamiento}

Trata-se de um estudo com delineamento transversal e comparativo, de abordagem quantitativa (Sampieri, Collado \& Lucio, 2013).

\section{Participantes}

Participaram do estudo 100 casais heterossexuais, com idade mínima de 18 anos, que estavam casados a pelo menos 1 ano, totalizando 200 participantes. A amostra foi acessada por conveniência, seguindo-se um efeito bola de neve, e foi estimada de acordo com os critérios propostos por Hair, Black, Babin, Andreson \& Thatam (2009), os quais consideram que o tamanho desejável da amostra compreenda de 15 a 20 sujeitos em cada grupo para cada variável independente considerada.

A idade média dos participantes do sexo masculino foi de $34,71(\mathrm{SD}=10,0)$ e do sexo feminino de 32,20 anos $(\mathrm{SD}=10,1)$. Quanto à escolaridade, houve variação entre ensino fundamental incompleto $(6,1 \%)$, ensino fundamental completo $(2,6 \%)$, ensino médio incompleto $(1,5 \%)$, ensino médio completo $(16,3 \%)$, ensino superior incompleto 
$(43,4 \%)$ e ensino superior completo $(30,1 \%)$. O tempo médio de relacionamento foi de 11 anos $(\mathrm{SD}=9,0)$. Referente ao estado civil, 33\% namoravam e $67 \%$ eram casados ou moravam juntos. Com relação a presença de filhos, $65 \%$ dos casais não os possuíam e os demais tinham filhos com até 5 anos $(11 \%)$, de 5 a 10 anos $(5 \%)$ e acima de $10 \operatorname{anos}(19 \%)$. Testes de associação entre as variáveis sociodemográficas mostraram que apenas a escolaridade diferiu significativamente em relação ao sexo $(p<0,002)$, indicando que as mulheres apresentavam níveis de escolaridade mais elevados que os homens.

\section{Instrumentos}

Ficha de Contato Inicial: desenvolvida pelas pesquisadoras para assegurar os critérios de inclusão da amostra (idade e tempo de relacionamento) e caracterizar os participantes quanto ao sexo, escolaridade e presença de filhos.

Questionário de Vinculação ao Pai e à Mãe (QVPM) (versão revista de Matos \& Costa, 2001): avalia as representações de vinculação com as figuras parentais em adultos, baseada nas contribuições de Bowlby $(1969,1973,1980)$, Ainsworth (1982, 1989, 1991; Ainsworth \& Bowlby, 1991) e Bartholomew (1990; Bartholomew \& Horowitz, 1991). O instrumento é composto por 30 itens avaliados através de uma escala do tipo Likert de 6 pontos (discordo totalmente a concordo totalmente), que avaliam a relação estabelecida com o pai e com a mãe, os quais são divididos em 3 subescalas, cada uma com 10 itens: inibição da exploração e da individualidade (avalia a percepção das limitações à expressão da individualidade e aos comportamentos exploratórios); qualidade do laço emocional (avalia a importância das figuras parentais como figuras de apego); e ansiedade de separação e dependência (avalia acerca das separações da figura de apego). Os dados obtidos nas três dimensões resultam em uma categoria final que indica um padrão de apego seguro (modelo positivo de si e dos outros, tendendo a ter relações de intimidade); desinvestido (modelo negativo dos outros, evitando relações afetivas e de intimidade); preocupado (modelo positivo dos outros e negativo de si, buscando constantemente a aceitação e aprovação dos outros); e amedrontado (modelo negativo de si e dos outros, caracterizado por uma reduzida autonomia e elevada dependência emocional). As propriedades psicométricas do questionário têm sido satisfatórias (Cordeiro, 2012; Gouveia, 2013; Matos, Barbosa, Almeida \& Costa, 1999). A subescala de inibição de exploração e atividade apresentou alfas de Cronbach que variaram de 0,85 a 0,95 para o pai e 0,85 a 0,93 para a mãe; a subescala de qualidade do laço emocional, apresentou alfas de 0,84 a 0,95 para o pai e 0,81 a 0,90 para a mãe, e, por fim, a subescala de ansiedade de separação e dependência, assumiu alfas de 0,80 a 0,87 para o pai e 0,76 a 0,85 para a mãe.

Questionário de Experiência em Relações Próximas (ERP) [tradução portuguesa de Moreira et al. (2015), da Experiences in Close Relationships Scale de Brennan, Clark \& Shaver (1998)]: o instrumento é composto por 36 perguntas divididas em duas subescalas, preocupação e evitação, para as quais os participantes indicam a forma como vivem a relação com seu parceiro/a, a partir de uma escala do tipo Likert de 3 pontos (discordo fortemente; neutro/misto; concordo fortemente). A subescala da preocupação contém 18 itens que se referem ao quanto o indivíduo se preocupa com o parceiro e com o relacionamento, bem como quanto ele necessita da proximidade física e emocional. A subescala evitação também contém 18 itens que abrangem o receio que o indivíduo tem da proximidade e da intimidade, a dependência, bem como a sensação de não poder confiar no outro quando necessário. As duas subescalas apresentaram índices satisfatórios de consistência interna (preocupação $\alpha=0,91$; evitação $\alpha=0,92$ ), o que indica boa qualidade psicométrica. Destaca-se que este instrumento tem sido um dos mais utilizados para avaliação de apego adulto (Natividade \& Shiramizu, 2015). 
Escala de Ajustamento Diádico Revista (EAD-R) [desenvolvida a partir da Dyadic Adjustment Scale (Spanier, 1976), adaptada da Escala de Ajustamento Diádico, desenvolvida por Busby, Christensen, Crane e Larson (1995) e traduzida por Hollist et al. (2012)]: a escala é composta por 14 itens, dividida em 3 subescalas: consenso, satisfação e coesão, que avaliam o ajustamento diádico. $\mathrm{O}$ consenso é composto por 3 dimensões (tomada de decisões, valores e afeto), a satisfação é constituída por duas dimensões (estabilidade e conflito), assim como a coesão (atividades e discussão). Considera-se que quanto maiores os escores nas subescalas, melhor a avaliação da qualidade conjugal (Hollist et al., 2012). Há, ainda, um resultado total que indica um relacionamento bem ajustado/sem sofrimento ou desajustado/com sofrimento. A EAD-R apresentou bons índices de consistência interna (consenso diádico $\alpha=0,81$; satisfação $\alpha=0,85$; coesão $\alpha=0,80$; e total $\alpha=0,90$ ), indicando boa qualidade psicométrica. Destaca-se que este é um dos instrumentos mais utilizados para avaliação da satisfação conjugal, inclusive no contexto brasileiro, sendo globalmente conhecida e com adaptações para diversos países (Pereira-Silva, Dessen \& Barbosa, 2015; Pereira-Silva, 2015; Peruchi, Donelli \& Marin, 2016; Scorsolini-Comin \& Santos, 2011).

\section{Procedimentos éticos e de coleta de dados}

Esse estudo foi avaliado e aprovado pelo Comitê de Ética em Pesquisa da Universidade do Vale do Rio dos Sinos (CAAE: 44099615.9.0000.5344), a fim de cumprir e garantir a adequação dos procedimentos éticos, conforme orienta a Resolução do Conselho Nacional de Saúde n 466/2012 (Brasil, 2012). Houve um encontro presencial com o casal ou um dos seus membros quando foi explicado o tema da pesquisa, bem como os instrumentos a serem preenchidos e a importância de assinarem o Termo de Consentimento Livre e Esclarecido (TCLE). Nesse momento, entregava-se um envelope fechado que constava dos TCLEs e dos questionários para que fossem respondidos em momento oportuno pelo casal separadamente. Todos foram orientados a devolverem para a pesquisadora os instrumentos descritos acima, devidamente preenchidos, dentro de um segundo envelope fechado e sem identificação, assegurando seu sigilo.

\section{Procedimentos de análise dos dados}

Os dados derivados dos instrumentos padronizados de pesquisa foram analisados quantitativamente, conforme orientação de seus manuais, por meio de estatísticas descritivas e inferenciais. Foram utilizadas as distribuições absoluta (n) e relativa (\%), bem como as medidas de tendência central e dispersão (média e desvio padrão) com o estudo de normalidade da distribuição dos dados pelo teste de Kolmorogov-Smirnov (correção de Lillifors).

A comparação das variáveis contínuas entre dois grupos independentes ocorreu pelo teste $t$, de Student ou de Mann Whitney, e para as variáveis categóricas foram utilizados os testes Exato, de Fisher, ou Qui-quadrado, de Pearson. Os dados foram analisados utilizando-se o SPSs for Windows (versão 20.0), e considerou-se o nível de significância de $\mathrm{p}<0,05$.

\section{Resultados}

Em relação ao padrão de apego primário, análises descritivas revelaram que $84,5 \%$ dos participantes tinham um padrão de apego seguro em relação ao pai e $79 \%$ em relação à mãe; $11,9 \%$ tinham um padrão de apego preocupado em relação ao pai e $15 \%$ em relação à mãe; e 3,6\%, um padrão de apego desinvestido em relação ao pai e $6 \%$ em relação à mãe. Nenhum dos participantes se caracterizou como tendo padrão de apego amedrontado. Também não houve diferença quanto ao padrão de apego primário entre homens e mulheres, conforme pode ser observado na tabela 1. 
Tabela 1

Padrão de apego [rimário referente ao pai e à mãe

\begin{tabular}{lrrrrrrr}
\hline $\begin{array}{c}\text { Apego } \\
\text { primário }\end{array}$ & $\begin{array}{c}\text { Total } \\
\text { amostra } \\
(\mathrm{N}=200)\end{array}$ & $\begin{array}{c}\text { Feminino } \\
(\mathrm{n}=100)\end{array}$ & $\begin{array}{c}\text { Masculino } \\
(\mathrm{n}=100)\end{array}$ & $p$ \\
\hline Apego - pai & $\mathrm{n}$ & $\%$ & $\mathrm{n}$ & $\%$ & $\mathrm{n}$ & $\%$ & 0,07 \\
\hline Seguro & 163 & 84,5 & 78 & 81,3 & 85 & 87,6 & \\
Preocupado & 23 & 11,9 & 16 & 16,7 & 7 & 7,2 & \\
Desinvestido & 7 & 3,6 & 2 & 2,1 & 5 & 5,2 & \\
Amedrontado & 0 & 0 & 0 & 0 & 0 & 0 & \\
\hline Apego - mãe & $\mathrm{n}$ & $\%$ & $\mathrm{n}$ & $\%$ & $\mathrm{n}$ & $\%$ & 0,17 \\
\hline Seguro & 158 & 79 & 77 & 77 & 81 & 81 & \\
Preocupado & 30 & 15 & 19 & 19 & 11 & 11 & \\
Desinvestido & 12 & 6 & 4 & 4 & 8 & 8 & \\
Amedrontado & 0 & 0 & 0 & 0 & 0 & 0 & \\
\hline
\end{tabular}

De acordo com os dados da tabela 2, constatou-se que no que diz respeito a comparação entre o padrão de apego primário e aquele estabelecido na relação conjugal, o Teste Exato, de Fischer, e o Qui-quadrado, de Pearson, revelaram associação significativa, tanto em relação ao padrão de apego constituído com a mãe quanto com o pai. O padrão de apego primário seguro relacionou-se com maiores níveis de preocupação e menores de evitação com o parceiro na relação conjugal. Já os padrões de apego primário preocupado e desinvestido associaram-se com maiores níveis de evitação e menores de preocupação com o parceiro.

Quanto ao padrão de apego primário e o ajustamento conjugal houve associação apenas com relação ao apego estabelecido com a mãe. Os resultados indicaram que o padrão de apego primário seguro está associado com um relacionamento bem ajustado e/ou sem sofrimento $(\mathrm{p}<0,04)$. Já os padrões de apego primário preocupado e desinvestido se associaram com relacionamentos desajustados e/ou com sofrimento $(\mathrm{p}<0,04)$, conforme pode ser observado na tabela 3 .

Considerando as dimensões de apego estabelecidas entre o casal e o ajustamento conjugal, o teste Qui-quadrado, de Pearson, revelou associações significativas entre os níveis de preocupação e evitação na relação com o parceiro. Relacionamentos com maiores níveis de evitação e menores de preocupação se associaram com relacionamentos desajustados e/ou com sofrimento $(\mathrm{p}<0,001)$. Por outro lado, menores níveis de evitação e maiores de preocupação se associaram com relacionamentos bem ajustados e/ou sem sofrimento $(\mathrm{p}<0,001)$.

Tabela 2

Padrão de apego primário referente ao pai e a mãe e dimensões do apego estabelecido na relação conjugal

\begin{tabular}{|c|c|c|c|c|c|c|c|c|c|c|}
\hline \multirow{3}{*}{ Apego primário } & \multicolumn{5}{|c|}{ Preocupação } & \multicolumn{5}{|c|}{ Evitação } \\
\hline & \multicolumn{2}{|c|}{ Menor $(n=46)$} & \multicolumn{2}{|c|}{ Maior $(n=154)$} & \multirow{2}{*}{$p$} & \multicolumn{2}{|c|}{ Menor $(\mathrm{n}=154)$} & \multicolumn{2}{|c|}{ Maior $(n=46)$} & \multirow{2}{*}{$p$} \\
\hline & $\mathrm{n}$ & $\%$ & $\mathrm{n}$ & $\%$ & & $\mathrm{n}$ & $\%$ & $\mathrm{n}$ & $\%$ & \\
\hline Apego - pai & & & & & 0,001 & & & & & 0,001 \\
\hline Seguro & 26 & 59,1 & 137 & 91,9 & & 137 & 91,9 & 26 & 59,1 & \\
\hline Preocupado & 15 & 34,1 & 8 & 5,4 & & 8 & 5,4 & 15 & 34,1 & \\
\hline Desinvestido & 3 & 6,8 & 4 & 2,7 & & 4 & 2,7 & 3 & 6,8 & \\
\hline Apego - mãe & & & & & 0,001 & & & & & 0,001 \\
\hline Seguro & 21 & 45,7 & 137 & 89 & & 137 & 89 & 21 & 45,7 & \\
\hline Preocupado & 18 & 39,1 & 12 & 7,8 & & 12 & 7,8 & 18 & 39,1 & \\
\hline Desinvestido & 7 & 15,2 & 5 & 3,2 & & 5 & 3,2 & 7 & 15,2 & \\
\hline
\end{tabular}


Tabela 3

Padrão de apego primário referente ao pai e a mãe e satisfação monjugal

\begin{tabular}{|c|c|c|c|c|c|}
\hline \multirow[t]{2}{*}{ Apego primário } & \multicolumn{2}{|c|}{$\begin{array}{c}\text { Relac. } \\
\text { ajustado } \\
(\mathrm{n}=141)\end{array}$} & \multicolumn{2}{|c|}{$\begin{array}{c}\text { Relac. } \\
\text { desajustado } \\
(\mathrm{n}=49)\end{array}$} & \multirow[t]{2}{*}{$p$} \\
\hline & $\mathrm{n}$ & $\%$ & $\mathrm{n}$ & $\%$ & \\
\hline Apego - pai & & & & & 0,19 \\
\hline Seguro & 118 & 86,8 & 45 & 78,9 & \\
\hline Preocupado & 15 & 11 & 8 & 14 & \\
\hline Desinvestido & 3 & 2,2 & 4 & 7 & \\
\hline Apego - mãe & & & & & 0,040 \\
\hline Seguro & 117 & 83 & 41 & 69,5 & \\
\hline Preocupado & 19 & 13,5 & 11 & 18,6 & \\
\hline Desinvestido & 5 & 3,5 & 7 & 11,9 & \\
\hline
\end{tabular}

Também houve relação entre a dimensão de consenso do instrumento que avalia o ajustamento conjugal com maiores níveis de preocupação e menores níveis de evitação ( $\mathrm{p}<0,005)$. Os dados são apresentados na tabela 4 .

No que diz respeito ao sexo, quando analisadas as dimensões de apego estabelecidas na relação conjugal, os resultados indicaram que tanto os homens como as mulheres tiveram maiores níveis de preocupação e menores níveis de evitação. No tocante ao ajustamento conjugal, também não houve diferença entre eles. Os dados constam na tabela 5. De modo semelhante, em relação às dimensões da satisfação conjugal, homens e mulheres apresentaram maiores níveis de satisfação e consenso e menores níveis de coesão. Os dados podem ser observados na tabela 6 .

Tabela 4

Dimensões do apego estabelecido entre o casal e a satisfação conjugal

\begin{tabular}{|c|c|c|c|c|c|c|c|c|c|c|}
\hline \multirow{3}{*}{ Apego casal } & \multicolumn{5}{|c|}{ Preocupação com parceiro } & \multicolumn{5}{|c|}{ Evitação do parceiro } \\
\hline & \multicolumn{2}{|c|}{ Menor $(n=46)$} & \multicolumn{2}{|c|}{ Maior $(n=154)$} & \multirow{2}{*}{$p$} & \multicolumn{2}{|c|}{ Menor $(n=154)$} & \multicolumn{2}{|c|}{ Maior $(n=46)$} & \multirow{2}{*}{$p$} \\
\hline & $\mathrm{n}$ & $\%$ & $\mathrm{n}$ & $\%$ & & $\mathrm{n}$ & $\%$ & $\mathrm{n}$ & $\%$ & \\
\hline Consenso & & & & & 0,005 & & & & & 0,005 \\
\hline Maior & 25 & 54,3 & 108 & 70,1 & & 108 & 70,1 & 25 & 54,3 & \\
\hline Menor & 18 & 39,1 & 26 & 16,9 & & 26 & 16,9 & 18 & 39,1 & \\
\hline Igual & 3 & 6,5 & 20 & 13 & & 20 & 13 & 3 & 6,5 & \\
\hline Satisfação & & & & & 0,09 & & & & & 0,09 \\
\hline Maior & 37 & 80,4 & 139 & 90,3 & & 139 & 90,3 & 37 & 80,4 & \\
\hline Menor & 8 & 17,4 & 11 & 7,1 & & 11 & 7,1 & 8 & 17,4 & \\
\hline Igual & 1 & 2,2 & 4 & 2,6 & & 4 & 2,6 & 1 & 2,2 & \\
\hline Coesão & & & & & 0,49 & & & & & 0,49 \\
\hline Maior & 12 & 26,1 & 35 & 22,7 & & 35 & 22,7 & 12 & 26,1 & \\
\hline Menor & 30 & 65,2 & 95 & 61,7 & & 95 & 61,7 & 30 & 65,2 & \\
\hline Igual & 4 & 8,7 & 24 & 15,6 & & 24 & 15,6 & 4 & 8,7 & \\
\hline Total & & & & & 0,005 & & & & & 0,005 \\
\hline Maior & 23 & 50 & 108 & 70,1 & & 108 & 70,1 & 23 & 50 & \\
\hline Menor & 21 & 45,7 & 33 & 21,4 & & 33 & 21,4 & 21 & 45,7 & \\
\hline Igual & 2 & 4,3 & 13 & 8,4 & & 13 & 8,4 & 2 & 4,3 & \\
\hline Total categoria & & & & & 0,001 & & & & & 0,001 \\
\hline Relac. ajustado & 22 & 47,8 & 119 & 77,3 & & 119 & 77,3 & 22 & 47,8 & \\
\hline Relac. desajustado & 24 & 52,2 & 35 & 22,7 & & 35 & 22,7 & 24 & 52,2 & \\
\hline
\end{tabular}


Tabela 5

Diferença entre homens e mulheres em relação às dimensões de apego estabelecidas entre o casal

\begin{tabular}{lccccc}
\hline & \multicolumn{2}{c}{ Feminino } & \multicolumn{2}{c}{ Masculino } & \\
\cline { 2 - 4 } Apego casal & $(\mathrm{n}=100)$ & \multicolumn{2}{c}{$(\mathrm{n}=100)$} & \\
\cline { 2 - 4 } & $\mathrm{n}$ & $\%$ & $\mathrm{n}$ & $\%$ & \\
\cline { 2 - 5 } & & & & & 0,74 \\
\hline Preocupação & & & & & \\
\hline Menor & 24 & 24 & 22 & 22 & \\
Maior & 76 & 76 & 78 & 78 & \\
\hline \multicolumn{1}{c}{ Evitação } & & & & & 0,74 \\
\hline Menor & 76 & 76 & 78 & 78 & \\
Maior & 24 & 24 & 22 & 22 & \\
\hline
\end{tabular}

Tabela 6

Diferença entre homens e mulheres em relação a satisfação conjugal

\begin{tabular}{|c|c|c|c|c|c|}
\hline \multirow{2}{*}{ Satisfação conjugal } & \multicolumn{2}{|c|}{$\begin{array}{l}\text { Feminino }(\mathrm{n} \\
=100)\end{array}$} & \multicolumn{2}{|c|}{$\begin{array}{l}\text { Masculino (n } \\
=100)\end{array}$} & \multirow{2}{*}{$p$} \\
\hline & $\mathrm{n}$ & $\%$ & $\mathrm{n}$ & $\%$ & \\
\hline Consenso & & & & & 0,53 \\
\hline Maior & 65 & 65 & 68 & 68 & \\
\hline Menor & 25 & 25 & 19 & 19 & \\
\hline Igual & 10 & 10 & 13 & 13 & \\
\hline Satisfação & & & & & 0,30 \\
\hline Maior & 85 & 85 & 91 & 91 & \\
\hline Menor & 11 & 11 & 8 & 8 & \\
\hline Igual & 4 & 4 & 1 & 1 & \\
\hline Coesão & & & & & 0,24 \\
\hline Maior & 26 & 26 & 21 & 21 & \\
\hline Menor & 64 & 64 & 61 & 61 & \\
\hline Igual & 10 & 10 & 18 & 18 & \\
\hline Total & & & & & 0,81 \\
\hline Maior & 67 & 67 & 64 & 64 & \\
\hline Menor & 25 & 25 & 29 & 29 & \\
\hline Igual & 8 & 8 & 7 & 7 & \\
\hline Total categoria & & & & & 0,64 \\
\hline Relac. ajustado & 69 & 69 & 72 & 72 & \\
\hline Relac. desajustado & 31 & 31 & 28 & 28 & \\
\hline
\end{tabular}

\section{Discussão}

O presente estudo teve como principal objetivo investigar a relação entre o padrão de apego primário (analisado através do QVPM) e as dimensões do apego estabelecido na relação conjugal (analisado através das dimensões do ERP), assim como a influência destes no ajustamento conjugal (analisado através do EAD-R). Observou-se que o padrão de apego primário seguro prevaleceu entre os participantes e também foi o mais presente entre aqueles que apresentaram maiores níveis de preocupação e menores de evitação com o seu parceiro/a na relação conjugal. A preocupação diz respeito a como o indivíduo se preocupa com o parceiro e com o relacionamento, e o quanto ele necessita da sua proximidade física e emocional. Já a evitação se refere ao maior desconforto com a intimidade, sendo características de indivíduos que preferem o distanciamento emocional ou, por outro lado, tendem a ser mais dependentes do parceiro (Brennan et al., 1998; Mikulincer \& Shaver, 2007; Moreira et al., 2015). Portanto, tais dados corroboram a literatura que aponta que os padrões de apego estabelecidos na infância tendem a se repetir nas relações futuras (Azevedo, 2013; Brennan et al., 1998; Feeney, 2008).

Bowlby (2004) afirmou que a capacidade de confiar nos outros, assim como a autoconfiança, são frutos de um ambiente seguro construído com as primeiras figuras de apego do indivíduo, o que encoraja a explorar o mundo e conhecer outras pessoas, acreditando que tem a quem recorrer em caso de necessidade. No relacionamento conjugal, pessoas com padrão de apego seguro e escores mais baixos de evitação tendem a confiar mais no parceiro, desenvolver maior intimidade, manifestar menos ciúmes e ter mais autonomia (Matos \& Costa, 2001).

Já o padrão de apego primário preocupado, que caracteriza indivíduos que estão sempre em busca da aprovação e aceitação dos demais, bem como o padrão de apego primário desinvestido, 
apresentado por indivíduos que preferem manter sua independência sem se envolver em relações de intimidade (Matos \& Costa, 2001; Mikulincer $\&$ Shaver, 2007), foram menos prevalentes entre os participantes e tiveram associação com maiores níveis de evitação nas relações conjugais. Tais padrões de apego são caracterizados por níveis mais elevados de ansiedade de separação, o que pode repercutir na construção de uma relação na qual o indivíduo depende mais do parceiro para realizar atividades, e tende a não se sentir confortável longe dele. Estudos indicam que indivíduos com níveis mais elevados de evitação podem entender pequenos sinais do parceiro como rejeição, como, por exemplo, atrasos a encontros e não responder a uma ligação, o que pode estar relacionado ao medo do abandono (Bastard, 2013; Pilkington \& Woods, 1999). Esses eventos podem gerar situações de estresse e ciúmes, que costumam distanciar o parceiro/a (Davis, Shaver \& Vernon, 2004; Sobral, Almeida \& Costa, 2010).

Os padrões de apego primário e os estabelecidos na relação conjugal também foram relacionados ao ajustamento conjugal. Os resultados indicaram uma associação entre o padrão de apego primário seguro e os relacionamentos bem ajustados e/ou sem sofrimento. Já os padrões de apego preocupado e desinvestido se associaram com relacionamentos mais desajustados e/ou com sofrimento. Tais dados permitem compreender que a dificuldade de individualização e a falta de autonomia e segurança repercutem negativamente nos relacionamentos conjugais (Melo \& Mota, 2013). Um relacionamento bem ajustado e/ou sem sofrimento se caracteriza por níveis mais elevados de consenso, satisfação e coesão. Costuma haver maior concordância na tomada de decisões e nos valores e coerência na resolução de conflitos, além de conseguirem conversar para trocar ideias e discutir atividades (Spanier, 1976), aspectos que remetem à confiança. Destaca-se que a confiança, que é parte da qualidade do apego, promove o desenvolvimento da intimidade e está fortemente relacionada à satisfação conjugal (Hazan \& Shaver, 1987).

O apego estabelecido na relação conjugal, avaliado pela preocupação e evitação em relação ao parceiro, também se mostrou associado ao ajustamento do casal. Níveis mais altos de preocupação e mais baixos de evitação quanto ao parceiro se associaram com relacionamentos bem ajustados e/ ou sem sofrimento. Por outro lado, maiores níveis de evitação e menores de preocupação se associaram a um relacionamento mais desajustado e/ou com sofrimento. O desconforto com a intimidade, característico da dimensão de evitação, pode fazer com que a autonomia prevaleça sobre a intimidade, levando a indisposição para discussão de problemas e expressão de sentimentos, fazendo com o que parceiro o perceba distante emocionalmente, o que pode justificar o desajustamento conjugal (Montoro, 2004; Semensato \& Bosa, 2013; Zeifman \& Hazan, 2008). Ainda, o consenso, caracterizado pela tomada de decisões, valores e afeto, teve associação com menores níveis de evitação e maiores de preocupação.

Por fim, quando comparado o padrão de apego primário entre homens e mulheres não se constatou diferenças. Para ambos o apego primário prevalente foi o seguro, assim como tiveram níveis mais altos de preocupação e mais baixos de evitação, possibilitando pensar que o apego estabelecido nas relações está mais relacionado com o apego que cada membro do casal estabeleceu com seus pais, especialmente sua mãe. Embora alguns estudos (Féres-Carneiro, 2001; Zordan, 2010) destaquem que os homens e as mulheres vivenciam o relacionamento de forma diversa, não houve diferença entre eles em relação ao ajustamento conjugal nesta pesquisa.

Em síntese, os resultados encontrados corroboram que o padrão de apego estabelecido a partir das experiências iniciais tende a se manter nos relacionamentos entre casais e tem influência no ajustamento conjugal. Conclui-se, portanto, que 
embora seja esperado que os indivíduos com padrões de apego preocupado ou desinvestido tendam a evitar a proximidade com as pessoas, não significa que eles não construam e mantenham relações conjugais. A questão parece estar centrada na qualidade da relação, uma vez que ela pode ser permeada por ciúmes, dependência, insegurança, medo do abandono e pouca intimidade (Barstad, 2013). Nesse sentido, faz-se importante que os profissionais da saúde que estão implicados no tratamento de casais tenham clareza sobre essa questão, podendo auxiliá-los na constituição de laços de afeto mais ajustados por meio da construção de um padrão de apego seguro entre eles.

\section{Considerações finais}

Em conjunto, os dados revelados apresentam contribuição importante no que se refere à dinâmica dos relacionamentos conjugais, especialmente no tocante a relação entre os padrões de apego primário e os estabelecidos na relação, indicando uma tendência à repetição que tem efeito sobre o seu ajustamento. Contudo, não se pode desconsiderar as limitações implicadas neste estudo que devem ter impactado os resultados encontrados. Por exemplo, sabe-se que há muitas variáveis que afetam o ajustamento conjugal, como tempo de relacionamento, presença de filhos, atração sexual, papeis sociais exercidos, entre outras, que não foram consideradas neste estudo. Além disso, devido a amostra ter sido acessada por conveniência, ela teve certa homogeneidade em sua caracterização, o que restringe os dados às suas especificidades: casais jovens, sem filhos e com nível médio a alto de escolaridade. Além disso, o fato de os instrumentos de pesquisa terem sido autoaplicados na ausência da pesquisadora não há como saber se o casal respondeu de forma independente e com atenção a todos os itens.

Para estudos futuros, sugere-se a realização de análises pareadas para avaliar os padrões de apego e o ajustamento conjugal, aumentando a eficiência do teste estatístico por torná-lo mais sensível às pequenas diferenças que possam haver entre os casais. Acredita-se que a realização de estudos longitudinais que permitam avaliar o padrão de apego primário na infância e compará-lo com o padrão estabelecido quando adultos na relação conjugal, também proporcionariam dados mais fidedignos em relação à repetição do comportamento, possibilitando maior compreensão sobre o fenômeno.

De qualquer forma, dentre as variáveis consideradas os resultados são relevantes, especialmente considerando que são poucos os estudos no contexto brasileiro que se dedicam a entender a associação entre os padrões de apego primário e estabelecido entre o casal e sua influência no ajustamento conjugal, a partir da perspectiva de homens e mulheres. Além disso, o conhecimento produzido poderá fundamentar intervenções sobre a conjugalidade no âmbito da clínica com casais ou programas de prevenção ou promoção de saúde que tenham esse público-alvo.

\section{Referências}

Ainsworth, M. D. S. (1982). Attachment: retrospect and prospect. New York: Basic Books.

Ainsworth, M. S. D. (1989). Attachment beyond infancy. American Psychology, 44, 709-716. DOI: 10.1023/A:1001944509409

Ainsworth, M. D. S. (1991). Attachments and other affectional bonds across the life cycle. Em C. M. Parkes, J. Stevenson-Hinde \& P. Marris (eds.), Attachment across the life cycle (pp. 33-51). London: Routledge.

Ainsworth, M. D. S., Blehar, M. C., Waters, E. \& Wall, S. (1978). Patterns of attachment: a psychological study of the strange situation. Hillsdale: Lawrence Erlbaum.

Ainsworth, M. S. \& Bowlby, J. (1991). An ethological approach to personality development. American Psychologist, 46(4), 333-341. DOI: 10.1037/0003-066x.46.4.333 
Attili, G. (2006). Apego e amor: entenda porque escolhemos nosso parceiro. J. A. Beraldin da Silva (trad.). São Paulo: Paulinas.

Azevedo, M. I. G. B. S. (2013). Vinculação em casais adultos e sua relação com os respetivos estilos de vinculação parental (Dissertação de mestrado). Universidade Lusófona de Humanidades e Tecnologias, Lisboa, Portugal.

Barstad, M. G. (2013). Do berço ao túmulo (Dissertação de doutorado). Pontifícia Universidade Católica do Rio de Janeiro, Rio de Janeiro, Brasil.

Bartholomew, K. (1990). Avoidance of intimacy: an attachment perspective. Journal of Social and Personal relationships, 7(2), 147-178. DOI: 10.1177/0265407590072001

Bartholomew, K. \& Horowitz, L. M. (1991). Attachment styles among young adults: a test of a four-category model. Journal of Personality and Social Psychology, 61(2), 226-244. DOI: 10.1037/0022-3514.61.2.226

Bogda, D. K. \& Sendil, G. (2012). Investigating infidelity tendency and conflict management based on attachment styles and gender. Elektronik Sosyal Bilimler Dergisi, 40(40), 205-219.

Bowlby, J. (1969). Attachment and loss: Vol 1. Attachment. New York: Basic Books.

Bowlby, J. (1973). Attachment and loss: Vol 2. Separation. New York: Basic Books.

Bowlby, J. (1980). Attachment and loss: Vol 3. Loss; sadness and depression. New York: Basic Books.

Bowlby, J. (1989). Uma base segura: Aplicações clínicas da teoria do apego. S. Monteiro de Barros (trad.). Porto Alegre: Artes Médicas.

Bowlby, J. (2004). Separação, angústia e raiva. São Paulo: Martins Fontes.

Bowlby, J. (2009). Apego: a natureza do vínculo. São Paulo: Martins Fontes.

Brennan, K. A., Clark, C. L. \& Shaver, P. R. (1998). Self-report measurement of adult attachment: an integrative overview. Em J. A. Simpson \& W. S. Rholes (eds.), Attachment theory and close relationships (pp. 46-76). New York: Guilford Press.

Bretherton, I. \& Munholland, K. A. (2008). Internal working models in attachment relationships: elaborating a central construct in attachment theory. Em J. Cassidy \& P. R. Shaver (eds.), Handbook of attachment: theory, research, and clinical applications (pp. 102-127). New York: Guilford Press.

Busby, D. M., Christensen, C., Crane, D. R. \& Larson, J. H. (1995). A revision of the Dyadic Adjustment Scale for use with distressed and nondistressed couples: construct hierarchy and multidimensional scales. Journal of Marital and Family Therapy, 21(3), 289-308. DoI: 10.1111/ j.1752-0606.1995.tb00163.x

Collins, N. L. \& Read, S. J. (1990). Adult attachment, working models, and relationship quality in dating couples. Journal of Personality and Social Psychology, 58, 644-663. DOI: 10.1037/00223514.58.4.644

Cordeiro, R. (2012). Vinculação e temperamento afectivo em jovens adultos (Dissertação de doutorado). Universidade de Lisboa, Lisboa, Portugal.

Crowell, J. A., Treboux, D. \& Waters, E. (2002). Stability of attachment representations: the transition to marriage. Developmental Psychology, 38(4), 467-479. DOI: 10.1037/0012-1649.38.4.467

Dalbem, J. X. \& Dell'Aglio, D. D. (2005). Teoria do apego: bases conceituais e desenvolvimento dos modelos internos de funcionamento. Arquivos brasileiros de psicologia, 57(1), 12-24.

Davis, D., Shaver, P. R. \& Vernon, M. L. (2004). Attachment style and subjective motivations for sex. Personality and Social Psychology Bulletin, 30(8), 1076-1090. DOI: 10.1177/0146167204264794

Feeney, J. A. (2008). Adult romantic attachment: developments in the study of couple relationships. Em J. Cassidy \& P. R. Shaver (eds.), Handbook of attachment: theory, research, and clinical 
applications (pp. 456-481). New York: Guilford Press.

Féres-Carneiro, T. (2001). Casamento contemporâneo: construção da identidade conjugal. Em T. Féres-Carneiro (ed.), Casamento e família: do social à clínica (pp. 67-80). Rio de Janeiro: Nau.

Fisher, J. \& Crandell, L. (2001). Patterns of relating in the couple. Em C. Clulow (ed.), Adult attachment and couple psychotherapy: the secure base in practice and research (pp. 15-27). New York/London: Routledge.

Fraley, R. C. (2002). Attachment stability from infancy to adulthood: meta-analysis and dynamic modeling of developmental mechanisms. Personality and Social Psychology Review, 6(2), 123-151. DOI: 10.1207/s15327957pspr0602_03

Fraley, R. C., Waller, N. G. \& Brennan, K. A. (2000). An item response theory analysis of self-report measures of adult attachment. Journal of personality and social psychology, 78(2), 350-365. DOI: 10.1037/0022-3514.78.2.350

Furman, W., Simon, V., Shaffer, L. \& Bouchey, H. A. (2002). Adolescent's working models and styles for relationships with parents, friends and romantic patners. Child Development, 73, 241-255. DOI: 10.1111/1467-8624.00403

Gouveia, M. J. C. (2013). Conflito interparental, vinculação aos pais e competências sociais do jovem adulto (Dissertação de mestrado). Universidade de Lisboa, Lisboa, Portugal.

Hair, J. F., Black, W. C., Babin, B. J., Andreson, R. E. \& Thatam, R. L. (2009). Análise multivariada de dados. Porto Alegre: Bookman.

Hazan, C. \& Shaver, P. (1987). Romantic love conceptualizes as an attachment process. Journal of Personality and Social Psychology, 3(52), 511-524. DOI: 10.1037/0022-3514.52.3.511

Hollist, C. S., Falceto, O. G., Ferreira, L. M., Miller, R. B., Springer, P. R., Fernandes, C. L. \& Nunes, N. A. (2012). Portuguese translation and validation of the Revised Dyadic Adjustment Scale. Journal of Marital and Family
Therapy, 38(s1), 348-358. DOI: $10.1111 / \mathrm{j}$. 1752-0606.2012.00296.x

Irish, S. J. \& Cassidy, J. (1997). Preescholer's attention and memory for attachment relevant information. Child Development, 63(6), 11431153. DOI: 10.1111/j.1467-8624.1997.tb01990.x

Klaus, M. H., Kennel, J. H. \& Klaus, P. (2000). Vínculo: construindo as bases para um apego seguro e para a independência. Porto Alegre: Artes Médicas.

Lima, G. Q. (2010). História de vida e escolha conjugal em mulheres que sofrem de violência doméstica (Dissertação de mestrado). Pontifícia Universidade Católica do Rio Grande do Sul, Porto Alegre, Brasil.

Matos, P. M., Barbosa, S., Almeida, H. M. \& Costa, M. E. (1999). Parental attachment and identity in Portuguese late adolescents. Journal of Adolescence, 22(6), 805-818. DOI: 10.1006/ jado.1999.0271

Matos, P. M. \& Costa, M. E. (2001). Questionário de vinculação ao pai e à mãe: Versão III. Manuscrito não-publicado. Faculdade de Psicologia e de Ciências da Educação da Universidade do Porto, Portugal.

Melo, O. \& Mota, C. P. (2013). Vinculação amorosa e bem-estar em jovens de diferentes configurações familiares. Psicologia em Estudo, 18(4), 587597. DOI: 10.1590/s1413-73722013000400002

Mikulincer, M. \& Shaver, P. R. (2007). Attachment in adulthood: structure, dynamics, and change. New York: Guilford Press.

Montoro, G. M. C. (2004). Amor conjugal e padrões de relacionamento. Em M. A. F. Vitale (ed.), Laços amorosos: Terapia de casal e psicodrama (pp. 101-133). São Paulo: Editora Ágora.

Moreira, H., Martins, T., Gouveia, M. J. \& Canavarro, M. C. (2015). Assessing adult attachment across different contexts: validation of the Portuguese version of the experiences in close relationships-relationship structures questionnaire. Journal of Personality Assessment, 97(1), 22-30. DOI: 10.1080/00223891.2014.950377 
Mosmann, C., Wagner, A. \& Féres-Carneiro, T. (2006). Qualidade conjugal: mapeando conceitos. Paidéia, 16(35), 315-325. DOI: 10.1590/ s0103-863x2006000300003

Natividade, J. C. \& Shiramizu, V. K. M. (2015). Uma medida de apego: versão brasileira da Experiences in Close Relationship Scale-Reduzida (ECR-R-Brasil). Psicologia USP, 26(3), 484-494. DOI: 10.1590/0103-656420140086

Orbach, S. (2007). Separated attachments and sexual aliveness: how changing attachment patterns can enhance intimacy. Attachment: New Directions in Relational Psychoanalysis and Psychotherapy, 1(1), 8-17.

Overall, N. C., Girme, Y. U., Lemay Jr., E. P. \& Hammond, M. D. (2014). Attachment anxiety and reactions to relationship threat: the benefits and costs of inducing guilt in romantic partners. Journal of Personality and Social Psychology, 106(2), 235. DOI: 10.1037/a0034371

Pereira-Silva, N. L. (2015). Estresse e ajustamento conjugal de casais com filho (a) com Síndrome de Down. Interação em Psicologia, 19(2), 297308. DOI: 10.5380/psi.v19i2.32747

Pereira-Silva, N. L., Dessen, M. A. \& Barbosa, A. J. G. (2015). Ajustamento conjugal: comparação entre casais com e sem filhos com deficiência intelectual. Psico-USF, 20(2), 297-308. DOI: 10.1590/1413-82712015200210

Peruchi, R. C., Donelli, T. M. S. \& Marin, A. H. (2016). Ajustamento conjugal, relação mãe-bebê e sintomas psicofuncionais no primeiro ano de vida. Quaderns de Psicología, 18(3), 55-67. DOI: https://doi.org/10.5565/rev/qpsicologia.1363

Pietromonaco, P. R. \& Barrett, L. F. (2000). The internal working models concept: what do we really know about the self in relation to others? Review of General Psychology, 4(2), 155-175. DOI: 10.1037//1089-2680.4.2.155

Pilkington, C. J. \& Woods, S. P. (1999). Risk in intimacy as a chronically accessible schema. Journal of Social and Personal Relationships, 16(2), 249263. DOI: $10.1177 / 0265407599162007$
Pistole, M. C. \& Arricale, F. (2003). Understanding attachment: beliefs about conflict. Journal of Counseling \& Development, 81(3), 318-328. DOI: 10.1002/j.1556-6678.2003.tb00259.x

Resolução no 466 de 2012 (12 de dezembro de 2012). Conselho Nacional de Saúde. Brasília: Distrito Federal.

Rosado, J. S. \& Wagner, A. (2015). Qualidade, ajustamento e satisfação conjugal: revisão sistemática da literatura. Pensando familias, 19(2), 21-33.

Salvatore, J. E., Sally, I., Kuo, C., Steele, R. D., Simpson, J. A. \& Collins, W. A. (2011). Recovering from conflict in romantic relationships a developmental perspective. Psychological Science, 22(3), 376-383. DOI: 10.1177/0956797610397055

Sampieri, R. H., Collado, C. F. \& Lucio, P. B. (2013). Metodologia de pesquisa. Porto Alegre: Editora Penso.

Scheeren, P., Delatorre, M. Z., Neumann, A. P. \& Wagner, A. (2015). O papel preditor dos estilos de apego na resolução do conflito conjugal. Estudos e Pesquisas em Psicologia, 15(3), 835-852. DOI: 10.12957/epp.2015.19415

Scorsolini-Comin, F. \& Santos, M. A. D. (2011). Ajustamento diádico e satisfação conjugal: correlações entre os domínios de duas escalas de avaliação da conjugalidade. Psicologia: Reflexão e Crítica, 24(3), 467-475.

Semensato, M. R. \& Bosa, C. A. (2013). O script de apego compartilhado no casal. Arquivos Brasileiros de Psicologia, 65(1), 138-151.

Sobral, M. P., Almeida, P. R. \& Costa, M. E. (2010). Medo da intimidade, vinculação e divórcio parental: um estudo com jovens adultos [resumo expandido]. Em Actas do VII Simpósio Nacional de Investigação em Psicologia (pp. 933-1009). Universidade do Minho, Portugal.

Spanier, G. B. (1976). Measuring dyadic adjustment: new scales for assessing quality of marriage and similar dyads. Journal of Marriage and the Family, 38, 15-28. DOI: 10.2307/350547

Sroufe, L. A., Egelend, B., Carlson, E. \& Collins, W.A. (2005). Placing early attachment experiences in 
development context: The Minnesota Longitudinal Study. Em K. E. Grossmann, K. Grossmann $\&$ E. Waters (eds.), Attachment from infancy to adulthood: the major longitudinal studies (pp. 48-70). New York/London: The Guilford Press.

Wang, C. C. D., King, M. L. \& Debernardi, N. R. (2012). Adult attachment, cognitive appraisal, and university students' reactions to romantic infidelity. Journal of College Counseling, 15(2), 101-116. DOI: 10.1037/cou0000161
Zeifmand. \& Hazan, C. (2008). Pair bonds as attachments: reevaluating the evidence. Em J. Cassidy \& P. R. Shaver (eds.), Handbook of attachment: theory, research, and clinical applications (pp. 436-455). New York: Guilford Press.

Zordan, E. P. (2010). A separação conjugal na contemporaneidade: Motivos circunstâncias e contextos (Dissertação de doutorado). Pontifícia Universidade Católica do RS, Porto Alegre, Brasil. 
